\title{
Gobernanza y capital social en el comanejo de recursos bentónicos en Chile: aportes del análisis de redes al estudio de la pesca artesanal de pequeña escala
}

\author{
Governance and social capital in the co-management of benthic \\ resources in Chile: contributions from a network analysis to the \\ study of small-scale artisanal fisheries
}

\author{
ANDRÉS MARÍN \\ Pontificia Universidad Católica de Chile \\ STEFAN GELCICH \\ Pontificia Universidad Católica de Chile
}

RESUMEN El comanejo o manejo colaborativo es considerado una institución relacional ya que pone el énfasis no sólo en la administración de los recursos propiamente tales, sino en las relaciones sociales que se establecen entre múltiples actores con diversos intereses en el territorio. Por esto, existe un creciente interés en su estudio desde el análisis de redes sociales (ARS) y el concepto afín de capital social. En este artículo presentamos dos aplicaciones del uso de ARs para comprender el comanejo, utilizando el sistema chileno de áreas de manejo y explotación de recursos bentónicos (AMERB) como caso de estudio. Específicamente se aplican herramientas del ARS para: I) describir y analizar la red de gobernanza que subyace el régimen de AMERB, y 2) explorar el rol del capital social de las organizaciones de pescadores en el funcionamiento y desempeño del comanejo. En base a las aplicaciones descritas en el artículo planteamos que el enfoque de redes sociales entrega 
una mirada promisoria y complementaria para el desarrollo y reformulación de políticas públicas más efectivas y con creciente participación de las comunidades locales.

PALABRAS CLAVE Sistemas socioecológicos costeros, pesca artesanal, gobernabilidad local.

ABSTRACT Co-management or collaborative management is considered to be a relational institution, since it places emphasis not only on the administration of resources as such, but also on the social relations between multiple actors with diverse interests in the territory. For this reason, growing interest exists in studying comanagement using social network analysis (SNA) and the related concept of social capital. In this article, we present two applications for the use of SNA to understand co-management, using the Chilean system of Management and Exploitation Areas for Benthic Resources (AMERB) as a case study. SNA tools are applied speci-fically to: I) describe and analyse the governance network underlying the amerb system, and 2) explore the role of fisher organizations' social capital in co-management functioning and performance. Based on the applications described in the article, we propose the social network approach as a promising and complementary standpoint for the development and reformulation of more effective public policy, with growing participation of local communities.

KEYWORDS Coastal socio-ecological systems, small-scale fishing, local governability.

\section{Introducción}

Las aproximaciones convencionales al manejo de los recursos naturales han sido inefectivas en asegurar la sustentabilidad de los ecosistemas marinos y las pesquerías. Ni las políticas de «comando y control» impuestas por el Estado (Holling y Meffe, I996), ni los sistemas basados en instrumentos de mercado (Litz, 1994) han sido capaces de comprender e incorporar adecuadamente una de las grandes complejidades que enfrentan la explotación y conservación de los mares, a saber, la realidad de sus usuarios locales. Inspiradas en el diagnóstico reduccionista de la «tragedia de los comunes» (Hardin, I968) —según la cual el destino inevitable de los recursos de libre acceso es ser sobreexplotados como consecuencia de la búsqueda del máximo beneficio individual 
y la desconsideración de los costos sociales-, gran parte de las políticas y regulaciones han buscado someter a los pescadores a la ley o transformarlos en propietarios privados. Al ignorar las particularidades sociales, económicas y culturales de los pueblos costeros, el resultado de estas soluciones ha sido muchas veces la profundización de los problemas o el surgimiento de nuevas dificultades (Castilla, I994; Schurman, I996; Ibarra, Reid y Thorpe, 2000).

En las últimas décadas se ha desarrollado una tercera vía que no descansa puramente ni el Estado ni el mercado. Esta consiste en el manejo colaborativo o comanejo, y se fundamenta en la capacidad de los usuarios de instaurar instituciones de manejo propias y autorregularse en el uso y explotación de los recursos (Wade, I987). En efecto, las relaciones y acciones de producción e intercambio de los productos de la tierra y el mar se encuentran arraigadas en sistemas de conocimiento, valores, normas y relaciones sociales (Jentoft, McCay y Wilson, I998; Berkes, Colding y Folke, 2000; Berkes, 2003; Pretty, 2003; Ther, 2008). Desde este punto de vista, la imagen del individuo egoísta (y depredador) se desdibuja en favor del individuo social (e integrado a su entorno). La institución del comanejo se caracteriza por la devolución (parcial o total) de poder de toma de decisiones por parte del Estado a los usuarios de los recursos, a la sociedad civil (Berkes, George y Preston, I99I). Entre las posibles ventajas del comanejo se cuentan la reducción de los costos de transacción y de fiscalización de las regulaciones, una mejor división de funciones y la creación de mecanismos de resolución de conflictos (Carlsson y Berkes, 2005). Ejemplos de comanejo existen en todo el mundo, en la administración de recursos hídricos, forestales y pesqueros (Borrini-Feyerabend y otros, 2004). Sin embargo, no existen recetas para el éxito del comanejo, ni modelos de talla única. El comanejo no es un punto de partida fijo sino el resultado de procesos sociales y ecológicos permanentes y dinámicos (Carlsson y Berkes, 2005). La práctica y teoría del comanejo se encuentra estrechamente relacionada al manejo y estudio de los sistemas socioecológicos, entendidos como el acoplamiento y coevolución de los subsistemas sociales y naturales (Berkes y Folke, I998; Berkes, Colding y Folkes, 2003). Se trata de entender y mejorar la adaptabilidad del ser-humano-en-la-naturaleza como forma de acercamiento a una sustentabilidad práctica.

Recientemente se ha desarrollado una línea de investigación que ha aplicado la teoría y los métodos del análisis de redes sociales (ARS) al estudio de los sistemas de manejo de recursos naturales (Bodin, Crona y Ernstson, 2006; Bodin y Crona, 2008; Carlsson y Sanstrom, 2008). En efecto, ciertos 
fenómenos y procesos sociales — como el poder y la influencia, la cooperación y el conflicto, la información y el conocimiento- que inciden en las oportunidades de desarrollo sustentable, no dependen tanto de los atributos de los sujetos individuales como de las relaciones que se establecen entre los actores en el territorio. Las personas, grupos y organizaciones, al interactuar, forman estructuras relativamente estables o redes, las que permiten u obstaculizan la movilización de recursos e información que son relevantes para la acción humana. El análisis de redes permite el estudio formal de esas relaciones sociales y la estructura social (Scott, 2000). En el campo del comanejo, por ejemplo, Bodin, Crona y Ernstson (2006) han analizado qué patrones estructurales de las redes (por ejemplo, centralidad, densidad y heterogeneidad) pueden potenciar, y cómo, la generación de capital social y así sostener sistemas sustentables de comanejo.

El presente artículo pretende mostrar dos aplicaciones empíricas del ARs en el estudio de un sistema de comanejo de pesca artesanal chilena, específicamente abordando el caso del sistema de áreas de manejo y explotación de recursos bentónicos (АMERB) de Chile. El artículo se sustenta en información primaria y secundaria, y busca exponer las potencialidades de este enfoque para la comprensión de dos aspectos fundamentales en los sistemas de manejo en general, y para las pesquerías en particular: I) describir y analizar el sistema AMERB en tanto una red de gobernanza e identificar las complejidades que involucra su funcionamiento; 2) medir la existencia y distribución del capital social de puente y escalera entre las organizaciones de pescadores y explorar el rol y el potencial explicativo de las redes sociales en los resultados de esta política de administración pesquera (correlación del capital social con variables de desempeño de las AMERB).

En la siguiente sección se presenta una breve reseña de la historia y evolución de la política de AMERB. Luego, en los métodos, se describe el área de estudio y se describe el diseño y la metodología empleados. Las secciones tercera y cuarta responden a los dos objetivos arriba indicados, a saber, las aplicaciones del ARs en el estudio de la gobernanza y del capital social. En la discusión y conclusiones ofrecemos algunas reflexiones sobre las potencialidades y desafíos del ARs en el estudio del comanejo de recursos naturales y de las pesquerías artesanales. 


\subsection{Caso de estudio: el sistema de áreas de manejo y explotación de recursos bentónicos}

A fines de la década de los ochenta se produjo en Chile una crisis de sobreexplotación de los recursos bentónicos, principalmente del recurso loco (Concholepas concholepas), impulsada en gran medida por políticas de fomento a las exportaciones y por una fuerte demanda de los mercados asiáticos (Gelcich y otros, 20I0). En concreto, las capturas totales de loco aumentaron de entre 2.000-6.000 toneladas anuales en la primera mitad de los setenta, hasta cifras superiores a las 20.000 toneladas anuales en la década del ochenta (Gelcich y otros, 20I0). El colapso de los recursos estuvo acompañado, además, de importantes externalidades sociales y económicas, a saber, altos niveles conflictividad entre los habitantes de las caletas y los miles de buzos que migraban de una región a otra en búsqueda de mejores bancos de locos (Meltzoff, Lichtensztejn y Slotz, 2002; Gelcich y otros, 2010). Intentos inmediatos del Estado por detener y controlar la sobreexplotación incluyeron medidas de «comando y control», como la veda total extractiva entre I989 y 1992 (Castilla, I994). Sin embargo, estas medidas sólo empeoraron la situación agudizando la crisis económica y social del sector (Castilla y Defeo, 200I; Meltzoff, Lichtensztejn y Slotz, 2002). La solución de más largo plazo consistió en una medida de administración pesquera que combinaría la asignación de derechos colectivos de uso territorial y un mecanismo de comanejo (Castilla, I994).

Las AMERB fueron institucionalizadas en la Ley de Pesca de I99 I y formalmente implementadas en I997, una vez que se promulgara su reglamento de funcionamiento. En este sistema, las organizaciones de pescadores artesanales (sindicatos, cooperativas o comunidades indígenas) solicitan a la Subsecretaría de Pesca (Subpesca) la asignación de derechos temporales de uso sobre un área definida del borde costero y los recursos allí existentes. La formalización de las AMERB descansa en la presentación de una línea de base y un plan de manejo elaborados por instituciones de asistencia técnica (como consultoras y universidades) contratadas por los pescadores. En base a estos estudios se definen las especies principales y las cantidades anuales que el Estado autorizará a los usuarios extraer, buscando respetar la capacidad natural de recuperación del ecosistema.

Las AMERB se implementaron primero en el centro del país (entre las regiones iv de Coquimbo y viII del Bío-Bío), luego en el norte (regiones I de Tarapacá, II de Antofagasta y III de Copiapó), y finalmente en el sur (regiones 
x de Los Lagos, XI de Aysén y XII de Magallanes). Actualmente existen más de 700 áreas decretadas en todo el país (Gelcich y otros, 2010). La producción total de las AMERB ascendió a I 5.9I9 toneladas en el año 20Io, siendo las principales especies extraídas el loco (Concholepas concholepas), la lapas (Fissurella spp.), el erizo (Loxechinus albus), el piure (Pyura chilensis), la macha (Mesodesma donacium, Venus antiqua) y variados tipos de algas (por ejemplo, Lessonia trabeculata) (Gelcich y otros, 2006; Gelcich y otros, 2010).

Las evaluaciones realizadas del funcionamiento de este sistema muestran resultados heterogéneos, dependiendo del énfasis que se le asigna a las distintas dimensiones de la pesquería. Algunos estudios han presentado el sistema como un modelo exitoso y promisorio (Castilla y Defeo, 200I; Hilborn, Orensanz y Parma, 2005; Schumann, 2007), mientras que otros han identificado ciertas deficiencias (Castilla y Gelcich 2006; González y otros, 2006; San Martín, Parma y Orensanz, 20Iо). Asimismo, diversos factores han sido indicados como determinantes del buen funcionamiento de las AMERB, incluyendo económicos y técnicos (Subpesca, 2005), organizacionales (Schumann, 2007) y biofísicos (Thiel y otros, 2007). Algunos autores han enfatizado también la existencia de beneficios no económicos que justifican el interés de los pescadores en esta medida (Gelcich y otros, 2005, Castilla y Gelcich, 2008). Si bien los estudios han indicado la participación de diversos actores en el sistema AMERB (Gelcich y otros, 2006; Schumann, 2010), sugiriendo la existencia de una red de comanejo, sólo recientemente se han medido estas relaciones de manera sistemática (Marín y Berkes, 20I0; Marín y otros, 20I2), buscando extraer aprendizajes que permitan retroalimentar la toma de decisiones y la adaptación de la política.

\section{Metodología}

\section{1 Área de estudio}

La recolección de información para la aplicación de ARS al sistema de AMERB se realizó durante el invierno de 2008 en dos regiones de la zona centro sur de Chile, la v de Valparaíso y la viII del Bío-Bío, cubriendo I6 y 22 organizaciones de pescadores, respectivamente (véase figura 3). El muestreo fue de tipo propositivo (no probabilístico), buscándose abarcar el mayor número de casos posibles en el tiempo disponible. La muestra obtenida representa el 64 y 50 por ciento del total de organizaciones participantes del sistema AMERB en el año 2008 en dichas regiones. 
Las regiones estudiadas poseen realidades contrastantes que justifican su selección con un fin comparativo. Mientras que la región de Valparaíso se caracteriza por albergar las oficinas de la Subsecretaría de Pesca (Subpesca) y ser el centro de la toma de decisiones en materia pesquera, la del Bío-Bío representa una de las más importantes para la actividad artesanal en cuanto al número de pescadores y a las capturas totales y bentónicas. Además, mientras que en la primera las caletas artesanales tienden a ser mayoritariamente urbanas y turísticas, en la segunda éstas se ubican en contextos rurales y con menor conectividad.

\subsection{Diseño general}

Esta investigación se sustenta en la teoría y los métodos del ARS (Scott, 2000; Hanneman y Riddle, 2005). En términos generales el análisis de redes consiste en el uso de matrices, sociogramas y un conjunto de medidas formales para el estudio de las estructuras sociales (Scott, 2000). Para explicar el diseño metodológico empleado es necesario describir los actores involucrados, las relaciones estudiadas y el modo como se recogieron los datos, así como la forma en que estas relaciones fueron procesadas y analizadas.

Los actores de nuestro ejercicio son en primera instancia las 38 organizaciones de pescadores artesanales participantes en el sistema AMERB en las regiones arriba descritas. Luego están aquellos actores que definimos como contrapartes de las anteriores en términos del funcionamiento del comanejo y que son parte del entorno organizacional de las organizaciones de pescadores. Entre ellos se incluyen instituciones públicas, privadas y otras organizaciones y asociaciones de la pesca artesanal (véase tabla 2). Estos actores contraparte del sistema fueron identificados a priori a partir de entrevistas exploratorias y de la revisión de documentos de política pública y de la literatura.

Las relaciones de estudio fueron definidas como los vínculos establecidos por la organización de pescadores con sus contrapartes para el desarrollo de las AMERB. Para recoger datos respecto de estas relaciones escogimos a los presidentes electos de las organizaciones como los informantes del estudio, en tanto representantes formales de éstas en temas como las AMERB. Se aplicó un cuestionario semiestructurado que contenía preguntas específicamente relacionales, entre otros ítemes (véase Marín, 2009). Las relaciones fueron abordadas en términos de su valencia y de su fuerza a través de las siguientes preguntas: a) « ¿Cómo definiría Ud. la participación de las siguientes instituciones en el 
desarrollo de su AMERB?», con las alternativas de respuesta "participación facilitadora», "obstaculizadora» o «ninguna participación»; b) «¿Qué grado de confianza siente Ud. respecto de las siguientes instituciones en lo que al manejo de su área se refiere?», a partir de una escala tipo Likert con las categorías de «mucha confianza», «suficiente», «poca» o «nada». ${ }^{1}$ En ambos casos se presentó o leyó a los entrevistados un listado con los actores arriba descritos como contrapartes del comanejo, y se dio la posibilidad de agregar otros actores no incluidos en la lista.

La información recogida fue almacenada y procesada bajo el modelo de una red bimodal. Las redes bimodales representan las relaciones entre dos conjuntos o modos de actores o entidades de distinta naturaleza en una matriz $n \times m$ (Wasserman y Faust, I994; Borgatti y Everett, 1997). En este caso concreto, como se representa en la figura I, el primer modo $n$ está constituido por nuestra unidad de análisis, a saber, las organizaciones de pescadores artesanales (las filas en la figura). El segundo modo $m$, en tanto, se compone de los actores e instituciones contrapartes de las anteriores en el funcionamiento de las AMERB (las columnas en la figura). A su vez es importante indicar que distinguimos las contrapartes que operan a la misma escala local que las organizaciones, con las que se establecen relaciones de puente (bridging) u horizontales, y aquellas que operan a otras escalas (regional y/o nacional), con las que las relaciones son de escalera (linking) o verticales. La figura I también representa el uso de cuatro matrices bimodales, con la misma estructura y los mismos actores, para expresar y analizar —a través de datos binarios- el tipo de relaciones existentes y su fuerza.

Entre las múltiples medidas utilizadas en el ARs, este estudio empleó la de centralidad de grado (degree centrality) como base para el análisis. La centralidad permite conocer la posición de los nodos en el interior de una red (Freeman, 2000; Sanz, 2003). La centralidad de grado, en particular, refleja la prominencia relativa de los puntos focales dentro de una red en base a su nivel de conectividad (Faust, I997; Hanneman y Riddle, 2005). Para redes bimodales, la centralidad de grado se refiere al número total de relaciones de cada actor, expresado como una proporción sobre el total de vínculos posibles (Borgatti y Everett, I997; Faust, I997). Así, por ejemplo, en base a la figura I se puede

I. La variable grado de confianza de las relaciones fue posteriormente dicotomizada, generándose una variable relaciones de confianza (uniendo «mucha» y «suficiente» confianza) y otra relaciones de desconfianza («poca» $y$ «nada» de confianza). 


\begin{tabular}{|c|c|c|c|c|c|c|c|}
\hline \multicolumn{8}{|l|}{ Desconfianza } \\
\hline \multicolumn{8}{|l|}{ Confianza } \\
\hline \multicolumn{8}{|c|}{ Obstaculización } \\
\hline \multirow{3}{*}{\multicolumn{2}{|c|}{ Facilitación }} & \multicolumn{6}{|c|}{ Contrapartes en el co-manejo } \\
\hline & & \multicolumn{2}{|c|}{$\begin{array}{c}\text { Misma escala } \\
(\sim \text { relaciones de puente })\end{array}$} & \multicolumn{4}{|c|}{$\begin{array}{c}\text { Otras escalas } \\
(\sim \text { relaciones de escalera })\end{array}$} \\
\hline & & 1) & 2) & 3) & $\ldots$ & 28) & Otra \\
\hline \multirow{5}{*}{ 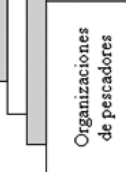 } & 1) & 1 & 0 & 0 & 1 & 0 & 0 \\
\hline & 2) & 0 & 1 & 0 & 0 & 1 & 0 \\
\hline & 3) & 0 & 0 & 1 & 0 & 0 & 0 \\
\hline & $\ldots$ & 1 & 1 & 1 & 0 & 0 & 1 \\
\hline & 38) & 0 & 0 & 0 & 0 & 1 & 0 \\
\hline
\end{tabular}

Figura 1. Cuatro aspectos de una red bimodal entre organizaciones de pescadores y actores contraparte en el sistema AMERB de comanejo

obtener el grado de centralidad de cada actor contraparte en la relación de facilitación atendiendo a las columnas de la primera matriz; y luego se pueden comparar los grados de los distintos actores. Esta es la lógica de análisis de la sección 3 de este capítulo, que utiliza el concepto de «nivel de facilitación neto» que cada actor contraparte obtuvo en el funcionamiento del comanejo para describir la red de gobernanza del sistema (véase tabla 2). Ahora bien, en base a la misma figura, si atendemos las filas de las matrices y calculamos la centralidad de grado de las organizaciones de pescadores, pueden analizarse y compararse el tamaño y la fuerza de las redes que cada organización posee. Esta es la lógica de análisis de la sección 4, en la que construimos índices (para integrar los distintos aspectos de las relaciones estudiadas) y adoptamos el concepto de capital social como punto de partida para investigar el posible efecto de esas redes sobre el desempeño del comanejo a nivel de las organizaciones. En particular, el enfoque egocéntrico del capital social consiste en el estudio de las redes desde la perspectiva parcial de los actores focales (o egos) en su relación con otros actores en su entorno (o alters) y el efecto sobre la consecución de sus metas (Van der Gaag y Webber, 2008; Lakon y otros, 2008).

\section{El sistema AMERB de comanejo como red de gobernanza}

Las conceptualizaciones del comanejo han avanzado desde entenderlo como un arreglo bipartito entre un Estado unitario y una comunidad homogénea, hacia caracterizaciones más inclusivas que incorporan múltiples actores de todos los sectores que operan a diversas escalas (Berkes, 2003; Mikalsen y Jentoft, 200I). Se ha criticado el énfasis en las estructuras formales de política pública, sugiriendo atender más los aspectos funcionales del comanejo como 
una asociación real y práctica (Carlsson y Berkes, 2005). En este sentido, la idea y la praxis del comanejo como una institución de reparto de poder ha evolucionado desde un modelo gubernamental y jerárquico hacia uno de gobernanza y de red. Esa visión considera que los múltiples vínculos y las relaciones sociales son la naturaleza misma del comanejo, y por tanto constituyen una red de gobernanza.

¿Qué podemos aprender sobre la gobernanza de un sistema de comanejo de recursos naturales, como el de las AMERB, a partir del ARs? De nuestra investigación se desprenden tres líneas de razonamiento. Primero, es posible analizar a los actores del sistema y comparar su contribución e importancia para el comanejo a partir de las relaciones establecidas con sus usuarios locales. La tabla I presenta los valores de centralidad de grado obtenidos por cada actor en cuanto a las relaciones de facilitación, obstaculización y facilitación neta, calculada como la diferencia de las anteriores (para mayores detalles de la operatoria, véase Marín y Berkes, 2010). Así, la observación de las dos primeras columnas sugiere que la gran mayoría de los actores son considerados como realizando aportes y obstaculizando el comanejo al mismo tiempo. No obstante, el promedio de los grados de centralidad de facilitación y obstaculización sugieren que hay preponderancia de las relaciones de facilitación en el sistema. La ordenación de los actores de acuerdo con el grado neto de facilitación proporciona la base para la identificación, a partir de los puntos de quiebre, de cuatro grupos de actores que, desde la mirada de los pescadores, juegan roles equivalentes de facilitación para funcionamiento de las AMERB: facilitación neta «alta», «media», «baja» y «negativa». También se observa que la diversidad de instituciones dentro de cada grupo (por ejemplo, estatales, sociedad civil y sector privado) y la variedad de funciones que desempeñan en el sistema (por ejemplo, la asignación de derechos, el conocimiento experto, y la comercialización), sugieren que ningún sector domina enteramente la red de comanejo.

En segundo lugar, la aplicación de ARs permite visualizar y analizar las posiciones de centralidad de los actores y sectores sociales en el sistema de acuerdo con las funciones que cumplen. La figura I presenta la imagen de la red del sistema AMERB construida a partir de datos empíricos y una clasificación de los actores definida por nosotros. En el centro se ubican las organizaciones de pescadores participantes del sistema AMERB; a su alrededor se presentan los actores contraparte del sistema, agrupados de acuerdo a la función principal que cumplen. El tamaño de los nodos (círculos, triángulos, cuadrados, etcétera) expresa el grado neto de facilitación de cada actor y el grosor de las líneas 


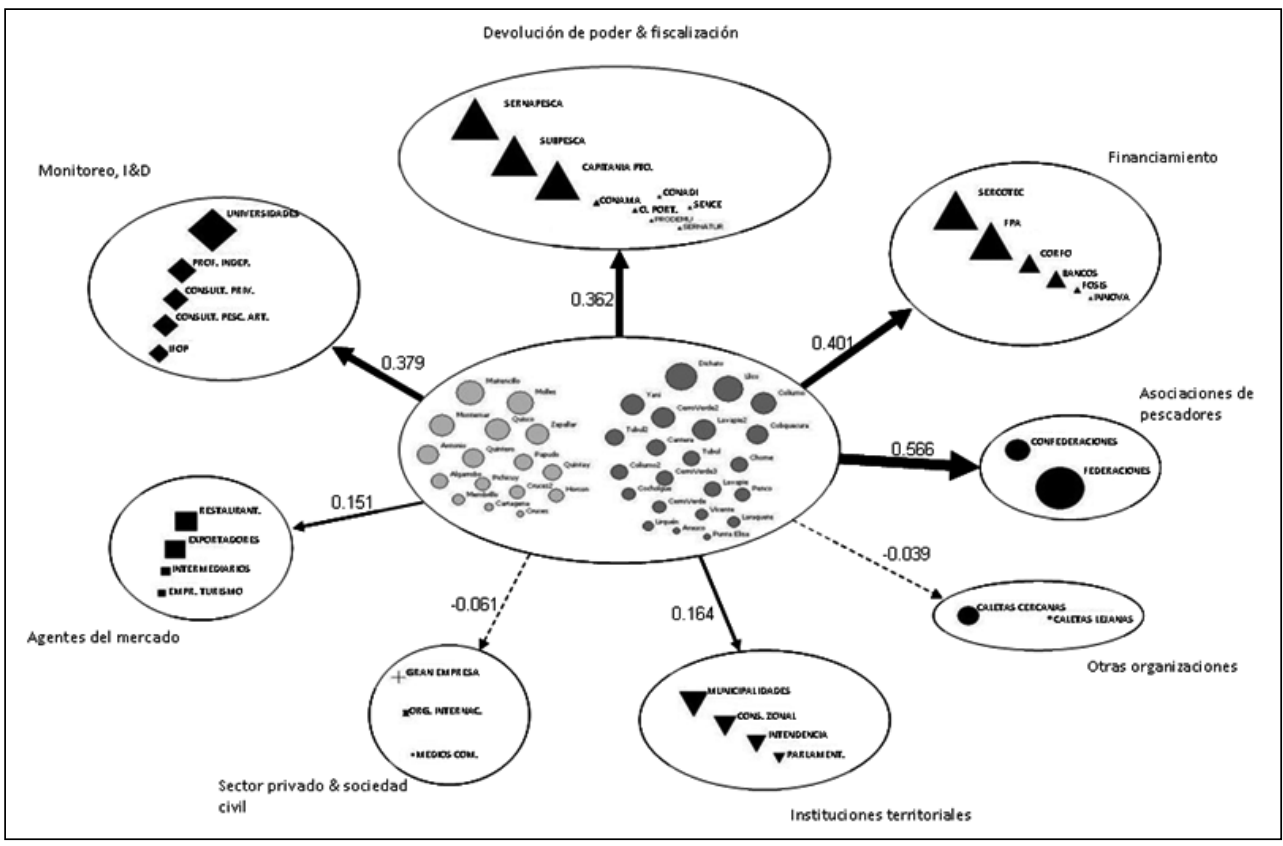

Figura 2. Red(es) de gobernanza en el sistema АмеRB desde la perspectiva de sus usuarios (adaptada de Marín y Berkes, 2010). Leyenda: tamaño de los nodos = grado neto de facilitación de los actores (in-out); grosor de las líneas = grado neto promedio de facilitación de los actores por cada grupo funcional; línea contínua = grado neto de facilitación positivo; línea punteada = grado neto de facilitación negativo.

el promedio de los grados de facilitación neta de los actores en cada grupo funcional (las líneas punteadas representan valores negativos). En la figura se puede observar, por ejemplo, la importancia central y el poder de las agencias del Estado dentro de la red de comanejo, en particular las que están a cargo de la asignación de AMERB y de financiamiento para su desarrollo. También la alta centralidad promedio de los actores que proveen asistencia técnica y monitoreo revela la importancia que posee para los usuarios el conocimiento experto para el funcionamiento del sistema. Por último, a la derecha del diagrama (al centro) se aprecia la alta apreciación de las relaciones establecidas con las asociaciones de pescadores artesanales en el desarrollo del comanejo, particularmente con las federaciones regionales. Posiblemente esto se explica por el rol que cumplen en la representación de los intereses sectoriales y en contrarrestar la influencia de otros actores privados sobre el ecosistema, y la del Estado. Esta situación contrasta, a su vez, con la baja vinculación y cooperación que se observa entre las organizaciones consultadas y otras caletas de pescadores cercanas y lejanas. 
TABLA 1. Valores de centralidad de grado de actores contraparte en el sistema AMERB

\begin{tabular}{|c|c|c|c|c|}
\hline & $\begin{array}{l}\text { Facilitación } \\
\text { (a) }\end{array}$ & $\begin{array}{l}\text { Obstaculización } \\
\text { (b) }\end{array}$ & $\begin{array}{l}\text { Facilitación neta } \\
\text { (a) - (b) }\end{array}$ & Grupos \\
\hline Federaciones regionales de la PA & 0.789 & 0.026 & 0.763 & \multirow{7}{*}{$\stackrel{\frac{D}{5}}{\frac{1}{D}}$} \\
\hline Universidades & 0.763 & 0.079 & 0.684 & \\
\hline Sercotec & 0.711 & 0.079 & 0.632 & \\
\hline Fondo de Fomento de la PA & 0.684 & 0.132 & 0.553 & \\
\hline Subpesca & 0.737 & 0.237 & 0.500 & \\
\hline Capitanía de Puerto & 0.711 & 0.211 & 0.500 & \\
\hline Sernapesca & 0.763 & 0.289 & 0.474 & \\
\hline Confederaciones nacionales de la PA & 0.395 & 0.026 & 0.368 & \multirow{12}{*}{$\frac{3}{\frac{3}{D}}$} \\
\hline Profesionales independientes & 0.421 & 0.053 & 0.368 & \\
\hline Consultoras dependientes de la PA & 0.368 & 0.026 & 0.342 & \\
\hline Municipio & 0.447 & 0.132 & 0.316 & \\
\hline Restaurantes & 0.342 & 0.053 & 0.289 & \\
\hline Concultoras privadas & 0.368 & 0.105 & 0.263 & \\
\hline Corfo & 0.316 & 0.053 & 0.263 & \\
\hline IFOP & 0.289 & 0.053 & 0.237 & \\
\hline Exportadores & 0.316 & 0.079 & 0.237 & \\
\hline Intendencia & 0.289 & 0.079 & 0.211 & \\
\hline Consejo Zonal de Pesca & 0.342 & 0.184 & 0.158 & \\
\hline Bancos & 0.289 & 0.132 & 0.158 & \\
\hline Fosis* & 0.079 & 0 & 0.079 & \multirow{11}{*}{$\stackrel{D}{\stackrel{D}{D}}$} \\
\hline Obras Protuarias* & 0.053 & 0 & 0.053 & \\
\hline Empresas de turismo & 0.105 & 0.053 & 0.053 & \\
\hline Organismos internacionales & 0.079 & 0.026 & 0.053 & \\
\hline Intermediarios & 0.132 & 0.105 & 0.026 & \\
\hline Sernatur* & 0.026 & 0 & 0.026 & \\
\hline Sence ${ }^{*}$ & 0.026 & 0 & 0.026 & \\
\hline Prodemu* & 0.026 & 0 & 0.026 & \\
\hline Medios de comunicación* & 0.026 & 0 & 0.026 & \\
\hline Innova* & 0.026 & 0 & 0.026 & \\
\hline Conadi* & 0.026 & 0 & 0.026 & \\
\hline Caletas cercanas & 0.342 & 0.342 & 0.000 & \multirow{7}{*}{ 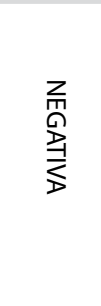 } \\
\hline ONG & 0.000 & 0.026 & -0.026 & \\
\hline Conama & 0.079 & 0.105 & -0.026 & \\
\hline Parlamentarios & 0.158 & 0.184 & -0.026 & \\
\hline Caletas lejanas & 0.053 & 0.132 & -0.079 & \\
\hline Grandes empresas & 0.211 & 0.421 & -0.211 & \\
\hline Promedio & 0.300 & 0.095 & 0.205 & \\
\hline
\end{tabular}

Leyenda: PA = pesca artsanal; * actores que no estaban en el listado original y que fueron agregados por los entrevistados; \# los actores se presentan ordenados de mayor a menor según el grado neto de facilitación. 


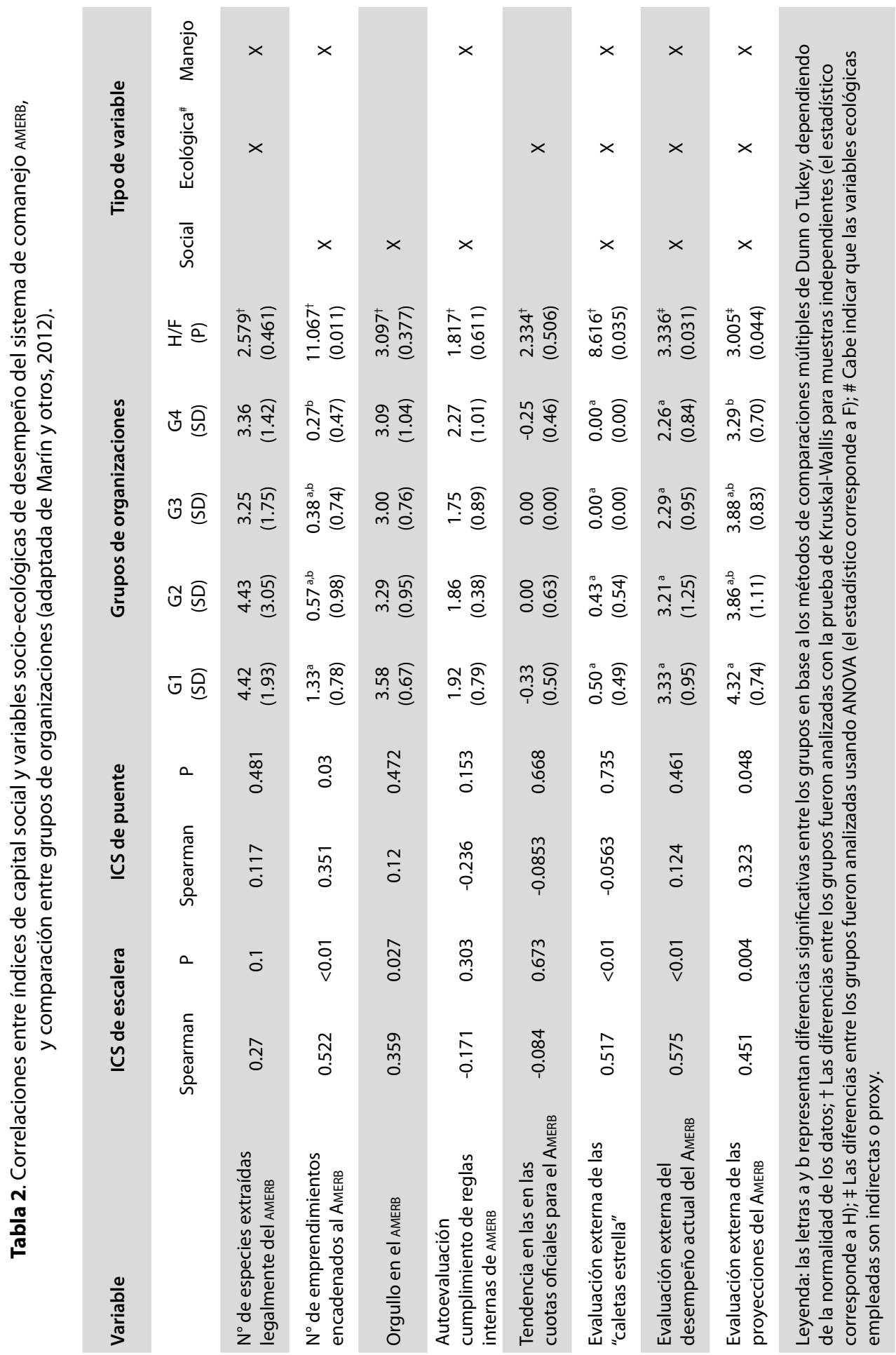




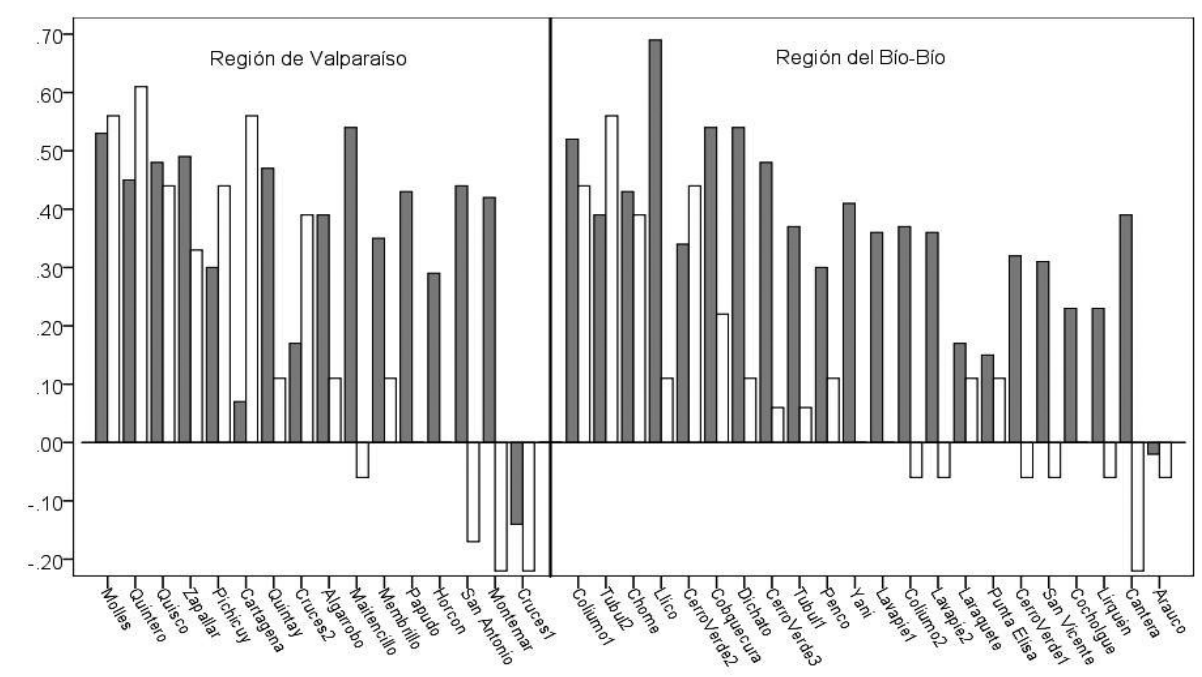

Figura 3. Capital social de escalera y puente de las organizaciones de pescadores artesanales participantes en el sistema AMERB en las regiones de Valparaíso y del Bío-Bío. Las barras grises representan el índice de capital social de escalera; las barras blancas representan el índice de capital social de puente; las organizaciones en cada región se presentan en orden decreciente a partir del valor promedio de ambos índices.

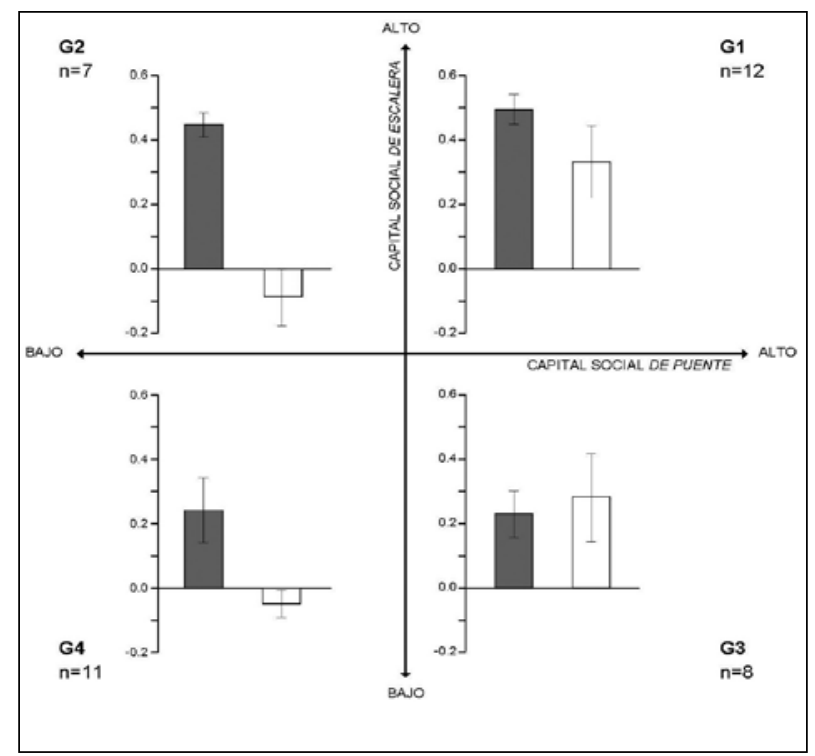

Figura 4. Tipología de organizaciones de pescadores según niveles de capital social de puente y escalera. Barras grises representan valores promedio de capital social de escalera; barras blancas representan valores promedio de capital social de puente (adaptada de Marín y otros, 2012). 
En síntesis, al agrupar a los actores de acuerdo con las funciones que desempeñan es posible observar que en la práctica el funcionamiento del comanejo implica múltiples redes de gobernanza. Es importante indicar que, aun cuando no se representa en la figura $\mathrm{I}$, estas redes no son siempre independientes entre sí. Muy por el contrario, el comanejo puede ser visto como una interacción dinámica entre estas múltiples redes funcionales. En este sentido, por lo general los desafíos y oportunidades de innovación y adaptación dentro del sistema emergen de los encuentros y desencuentros entre ellas y sus actores (véase Marín y Berkes, 2010).

\section{Capital social para comprender el funcionamiento de un sistema socioecológico de comanejo}

En la sección anterior se presentó la imagen del sistema AMERB como una red de comanejo diversa, extensa y compleja. En esta sección presentaremos la manera en que el ARs puede contribuir en la comprensión del funcionamiento de los sistemas socioecológicos. La pregunta orientadora es: ¿cómo el nivel de capital social de las organizaciones participantes en una red de manejo permite explicar los distintos niveles de éxito en el sistema? Esta aproximación reviste especial interés considerando que múltiples estudios (Pretty y Ward, 200I; Grafton, 2005; Gutiérrez y otros, 20I I) han identificado el capital social como un factor crítico en el desempeño de organizaciones y actores locales sobre el manejo de recursos naturales renovables.

El capital social ha sido definido de maneras diversas. Las definiciones, en general, mezclan la existencia de redes, confianzas, normas y reciprocidad entre miembros de la sociedad (Bourdieu, I985; Coleman, I988; Putnam, 2000). Pero la promesa central del capital social común a todas las definiciones es que la inversión de las personas y colectivos en relaciones sociales se verá retribuida en otras formas de capital y en el consecuente aumento en sus oportunidades y capacidades para lograr sus metas (Portes, I998). Esta hipótesis se ha hecho extensiva también al ámbito del manejo de los recursos naturales y la sustentabilidad ambiental (Pretty y Ward, 200I; Ballet, Sirven y Requiers-Desjardins, 2007). En consecuencia, mayores niveles de capital social (conectividad) estarían asociados a un mejor desempeño en el manejo de sistemas socioecológicos. Para poner a prueba esta hipótesis utilizando el ARs presentado anteriormente, nos focalizamos en las relaciones entre los grupos en el mismo nivel jerárquico, denominado capital social de puente, 
y los vínculos verticales de las organizaciones con los actores e instituciones más allá del nivel de la comunidad, denominado capital social de escalera (véase Woolcock, 200I; Arriagada, Miranda y Pavez, 2004; figura I). En base a datos empíricos, calculamos un índice para cada tipo de capital social y lo asociamos con un conjunto de variables de desempeño de manejo (Marín y otros, 20I2).

El índice de capital social de escalera (ICSE) captura el conjunto de conexiones de cada organización con los actores en otras escalas, y se compone de tres elementos: a) grado neto de facilitación (facilitación-obstaculización); b) grado neto de confianza (confiabilidad-desconfianza); c) heterogeneidad de los diferentes grupos funcionales. La heterogeneidad de los actores en una red puede ser un factor clave de un buen desempeño en redes de cooperación debido a la variedad de recursos que pueden proporcionar (Carlsson y Sandström, 2008). La puntuación ICSE para cada organización va entre I y o, y fue calculada como la media simple de los tres componentes indicados. Por su parte, el índice de capital social de puente (ICSP) representa los vínculos horizontales entre organizaciones del mismo nivel y fue calculado como la media entre: a) y b) según lo descrito anteriormente para ICSE pero basado en las relaciones horizontales entre grupos (caletas) y c) un factor complementario que representa características particulares de las interacciones entre grupos. Los lazos de puente han sido considerados importantes como factor que gatilla la acción colectiva (Krishna, 2002; Carlsson y Sandström, 2008).

Al calcular los índices se puede observar una distribución heterogénea de capital social de puente y escalera entre las diferentes organizaciones estudiadas (figura 3). Tanto para el ICSE como para el ICSP los valores van desde o, 6 a cifras negativas. Niveles de capital social alto representan organizaciones en las que las relaciones con otras organizaciones pueden potenciar una facilitación al acceso a recursos. Los resultados negativos de capital social representan situaciones en las que las relaciones de obstaculización y desconfianza sobrepasan aquellas de facilitación y confianza, determinando situaciones de conflicto y de acceso limitado a recursos importantes para el comanejo por parte de los usuarios.

El establecer índices de capital social, como los desarrollados aquí, permite poner a prueba la existencia de relaciones entre capital social de puente y escalera y el desempeño socioecológico de las organizaciones que co-manejan sus recursos. En nuestro trabajo, por ejemplo, los resultados de estas correlaciones (tabla 2) indican que el ICSE se correlaciona positivamente con cinco variables 
de distinto tipo. ${ }^{2}$ El índice de correlación más elevado se encuentra representado por la relación entre ICSE y la variable 'evaluación externa del desempeño actual del AMERB' ( $p=0.575 ; \mathrm{P}<0.000 \mathrm{I})$. Correlaciones significativas también se encuentran entre ICSE, el 'número de emprendimientos encadenados al AMERB' ( $\mathrm{p}=$ 0.522; P < 0.000I) y la 'evaluación externa de las «caletas estrella»' $(\mathrm{p}=0.5 \mathrm{I} 7$; $\mathrm{P}<0.000 \mathrm{I})$. En contraste, al analizar la asociación entre ICSP y el desempeño de AMERB sólo se encuentran relaciones significativas para el 'número de emprendimientos encadenados al AMERB' ( $\mathrm{p}=0.35 \mathrm{I} ; \mathrm{P}=0.03$ ) y la 'evaluación externa de las proyecciones futuras del AMERB' ( $\mathrm{p}=0.323 ; \mathrm{P}=0.048$ ). En términos generales, se observa que el tamaño y la fuerza de las relaciones de comanejo de las organizaciones tienen relación con ciertas variables de desempeño. Las organizaciones mejor conectadas tienden a tener un mejor desempeño.

Ahora bien, a un nivel más detallado, es posible que una misma organización posea distintos niveles de ICSE y de ICSP simultáneamente. En base a las medianas de los rangos de valores observados en ambos índices proponemos una tipología de cuatro grupos (figura 4): grupo I (GI): doce organizaciones con los valores más altos en ICSE y ICS; grupo $2\left(\mathrm{G}_{2}\right)$ : siete organizaciones con altos niveles de ICSE pero bajos puntajes de ICSP; grupo $3\left(\mathrm{G}_{3}\right)$ : ocho organizaciones con bajo ICSE pero alto ICSP; grupo $4\left(\mathrm{G}_{4}\right)$ : once organizaciones con bajos puntajes en ambos índices. Esta representación de los resultados permite una mejor comprensión de la heterogeneidad de los casos particulares. Adicionalmente, usando la prueba de Kruskal-Wallis y ANOva se pudo detectar diferencias significativas entre los grupos con respecto a cuatro de las variables de desempeño analizadas (véase tabla 2). Es interesante constatar nuevamente que las organizaciones con altos niveles de capital social de puente y escalera, aunque particularmente del primer tipo, tienden a ser las mejor evaluadas en términos socioecológicos (usando métodos de comparaciones múltiples de Dunn o Tukey). Las «caletas estrella» pueden no tener altos niveles de capital social de puente $\left(G_{2}\right)$, pero siempre poseen altos niveles de capital social de escalera (GI y G2).

\section{Discusión y conclusiones}

En este artículo hemos querido mostrar dos aplicaciones empíricas del ARS para el estudio de un sistema de comanejo en la pesca artesanal chilena. Co-

2. Para una descripción detallada de estas variables, sus fuentes, escalas de medición y su supuesta relación con la existencia de redes sociales, véase Marín y otros, 20I 2. 
rresponde ahora discutir sus potencialidades, limitaciones y oportunidades para avanzar en sus aportes.

El uso de una metodología sistemática, como es el ARs, puede servir a propósitos descriptivos y explicativos en el estudio de sistemas socioecológicos. En particular, la combinación de medidas numéricas y sociogramas (en este caso en una forma bimodal) permite representar y describir la multiplicidad y complejidad de relaciones que subyacen un sistema de comanejo. La imagen presentada aquí de las AMERB es congruente con las definiciones conceptuales actuales que conciben el comanejo como una red de gobernanza que va más allá de la potestad del Estado. La visualización de esta red entrega elementos para el análisis e identificación de posibles fortalezas y debilidades de sistemas de manejo.

Por su parte, la aproximación egocéntrica al capital social permite afinar el análisis desde una mirada macro a las realidades micro, desde el sistema de comanejo a las particularidades de sus usuarios locales. También permite calcular índices que contribuyen a establecer la importancia diferenciada de las relaciones verticales y horizontales de comanejo en pesquerías de pequeña escala. Además, provee una base para correlacionar cuantitativamente dimensiones sociales y ecológicas del manejo de recursos naturales renovables. El análisis presentado aquí es consecuente con la teoría, sugiriendo que las relaciones sociales y la conectividad son un factor concurrente (no determinante) del manejo sustentable de los recursos. En definitiva, el enfoque de redes del capital social ofrece una herramienta concreta para el análisis integrado de sistemas socioecológicos.

El uso de ARs para estudiar sistemas de manejo de recursos naturales costeros (u otros) presenta también desafíos. Entre ellos podemos indicar: I) la importancia de incorporar variables biológicas más complejas en el análisis, mediciones que dependen de información inexistente y que es costosa de recoger; 2) la oportunidad de integración en los análisis de un tercer tipo de capital social, a saber el capital social de unión (bonding) que refiere a las relaciones sociales internas entre los miembros de una misma organización; 3) la relevancia de realizar estudios longitudinales con el fin de identificar procesos de cambio y adaptación en las redes y en los sistemas socioecológicos; 4) finalmente, el desafío de contribuir a la toma de decisiones y al mejoramiento de los mecanismos de manejo a través de investigaciones aplicadas y con resultados relevantes para la política pública. 


\section{Referencias}

Arriagada, I., Miranda, F., y Pavez, T. (2004). Lineamientos de acción para el diseño de programas de superación de la pobreza desde el enfoque del capital social. Guía conceptual y metodológica. Serie Manuales, nro. 36, LC/L.2 I79-P, Santiago de Chile, Comisión Económica para América Latina y el Caribe (Cepal). Publicación de las Naciones Unidas.

Ballet, J., Sirven, N., y Requiers-Desjardins, M. (2007). Social Capital and Natural Resource Management: A Critical Perspective. The Journal of Environment Development, I6: 355-74.

Berkes, F., y Folke, C. (I998). Linking Social and Ecological Systems: Management Practices and Social Mechanisms for Building Resilience. Cambridge: Cambridge University Press.

Berkes, F. (2003). Alternatives to Conventional Management: Lessons from Small-Scale Fsheries. Environments, 3 I: 5-I9.

Berkes, F., George, P., y Preston, R. (I99i). Co-management: The Evolution of the Theory and Practice of Joint Administration of Living Resources. Alternatives, I 8 (2): I 2-I 8.

Berkes, F., Colding, J., y Folke, C. (2000). Rediscovery of Traditional Ecological Knowledge as Adaptive Management. Ecological Applications, ıо (5): I $25 \mathrm{I}-62$.

-. (2003). Navigating Social-Ecological Systems. Building Resilience for Complexity and Change. Cambridge: Cambridge University Press.

Bodin, Ö., y Crona, B. I. (2008). Management of Natural Resources at the Community Level: Exploring the Role of Social Capital and Leadership in a Rural Fishing Community. World Development, 36 (I2): 2763-79.

Bodin, Ö., Crona, B., y Ernstson, H. (2006). Social Networks in Natural Resource Management: What is There to Learn from a Structural Perspective? Ecology and Society, I I (2): r2. Disponible en <http://www.ecologyandsociety.org/voli I /iss2/resp2/>.

Borgatti, S., y Everett, M. (I997). Network Analysis of 2-mode Data. Social Networks, I9: 243-69.

Borrini-Feyerabend, G., Pimbert, M., Farvar, M. T., Kothari, A., y ReNARD, Y. (2004). Sharing Power. Learning by Doing in Co-management of Natural Resources Throughout the World. IIED and IUCN/CEESP/CMWG, Cenesta, Teherán, Irán.

Bourdieu, P. (I985). The Forms of Capital. En J. G. Richardson (editor), 
Handbook of Theory and Research for the Sociology of Education (24I58). Nueva York: Greenwood.

Carlsson, L., y Berkes, F. (2005). Co-management: Concepts and Methodological Implications. Journal of Environmental Management, 75: 65-76.

Carlsson, L., y Sandström, A. (2008). Network Governance of the Commons. International Journal of the Commons, 2 (I): 33-54. Disponible en $<$ http://www.thecommonsjournal.org/index.php/ijc/article/view/20>.

Castilla, J. C., y Defeo, O. (200I). Latin American Benthic Shellfisheries: Emphasis on Co-management and Experimental Practices. Reviews in Fish Biology and Fisheries, I I: I-30.

Castilla, J. C., y Gelcich, S. (2006). Chile: Experience with Management and Exploitation Areas for Coastal Fisheries as Building Blocks for LargeScale Marine Management. In Scaling up Marine Management: The Role of Marine Protected Areas (45-57). Washington: The World Bank.

-. (2008). Management of the Loco (Concholepas concholepas) as a Driver for Self-governance of Small-scale Benthic Fisheries in Chile. En R. Townsend, R. Shotton, y H. Uchida (eds.), Case Studies in Fisheries Self-governance (44I-45I). FAO Fisheries Technical paper 504. Roma: Food and Agriculture Organization of the United Nations.

Castilla, J. C. (I994). The Chilean Small Scale Benthic Shellfisheries and the Institutionalization of New Management Practices. Ecological International Bulletin, 21: 47-63.

Coleman, J. S. (1988). Social Capital in the Creation of Human Capital. American Journal of Sociology, 94: 95-I2I.

Faust, K. (1997). Centrality in affiliation networks. Social Networks, I9: I 57-9I. Freeman, L. (2000). Centrality in Social Networks: Conceptual Clarifications. Social Networks, I: 21 5-39.

Gelcich, S., Edwards-Jones, G., Kaiser, M. J., y Watson, E. (2005). Using Discourses for Policy Evaluation: The Case of Marine Common Property Rights in Chile. Society and Natural Resources, I 8: 377-9I.

Gelcich, S., Edwards-Jones, G., Kaiser, M. J., y Castilla, J.C. (2006). Co-management Policy Can Reduce Resilience in Traditionally Managed Marine Ecosystems. Ecosystems, 9: 95 I-66.

Gelcich, S., Hughes, T. P., Olsson, P. , Folke, C., Defeo, O., Fernández, M., Foale, S., Gunderson, L. H., Rodríguez-Sickert, C., Scheffer, M., Steneck, R. S., y Castilla, J. C. (20I0). Navigating Transformations in Governance of Chilean Marine Coastal Resources. PNAS, IO7 (39): I6794-99. 
González, J., Stotz, W., Garrido, J., Orezanz, J. M., Parma, A., Tapia, C., y Zuleta, A. (2006). The Chilean TURF System: How is it Performing in the Case of the Loco Fishery? Bulletin of Marine Science, 73 (3): 499-527.

Grafton, R. Q. (2005). Social Capital and Fisheries Governance. Ocean and Coastal Management, 48: 753-66.

Gutiérrez, N., Hilborn, R., y Defeo, O. (201 I). Leadership, Social Capital and Incentives Promote Successful Fisheries. Nature, 470: $386-89$.

Hanneman, R. A., y Riddle, M. (2005). Introduction to Social Network Methods. Riverside: University of California. Disponible en <http://faculty. ucr.edu/ hanneman/>.

Hardin, G. (I968). The Tragedy of the Commons. Science, I62: I243-48.

Hilborn, R., Orensanz, J. M., y Parma, A. M. (2005). Institutions, Incentives and the Future of Fisheries. Phil. Trans. R. Soc. B., 360: 47-57.

Holling, C. S., y Meffe, G. K. (I996). Command and Control and the Pathology of Natural Resource Management. Conservation Biology, Iо (2): 328-37.

Ibarra, A., Reid, C., y Thorpe, A. (2000). Neo-liberalism and its Impact on Overfishing and Overcapitalisation in the Marine Fisheries of Chile, Mexico and Peru. Food Policy, 25: 599-622.

Jentoft, S., McCay, B. J., y Wilson, D. C. (I998). Social Theory and Fisheries Comanagement. Marine Policy, 22 (4-5): 423-36.

KrishnA, A. (2002). Enhancing Political Participation in Democracies: What is the Role of Social Capital? Comparative Politicas Studies, 35 (4): 437-60.

Lakon, C. M., Godette, D., Dionne, C., Hipp, J. R. (2008). Network-based Approaches for Measuring Social Capital. En I. Kawachi, S. V. Subramanian y D. Kim (eds.), Social Capital and Health (63-8I). Nueva York: Springer.

Litz, F. T. (1994). Harnessing Market Forces in Natural Resources Management: Lessons from the Surf Clam Fishery. Boston College Environmental Affairs Law Review, 2I: 335-6r. Disponible en <http://lawdigitalcommons. bc.edu/ealr/vol2 I/iss2/ıo>.

Marín, A., y Berkes, F. (20IO). Network Approach for Understanding Smallscale Fisheries Governance: the Case of the Chilean Coastal Co-management System. Marine Policy, 34: 85 I-58.

Marín, A. (2009). Networks and Co-management in Small-scale Fisheries in Chile. Master's thesis. Natural Resources Institute, University of Manitoba. Marín, A., Gelcich, S., Castilla, J. C., y Berkes, F. (2OI2). Exploring Social Capital in Chile's Coastal Benthic Co-management System Using a Network Approach. Ecology and Society, I7 (I): I3. 
Meltzoff, S., Lichtensztajn, Y., y Stotz, W. (2002). Competing Visions for Marine Tenure and Co-management: Genesis of a Marine Management area System in Chile. Coastal Management, (30): 85-99.

Mikalsen, K. H. y Jentoft, S. (200I). From User-groups to Stakeholders? The Public Interest in Fisheries Management. Marine Policy, 25: $28 \mathrm{I}-92$.

Portes, A. (1998). Social Capital: its Origins and Applications in Modern Sociology. Annual Review of Sociology, 24: I-24. Disponible en <http://www. jstor.org/stable/223472>.

Pretty, J., y Ward, H. (200I). Social Capital and the Environment. World Development, 29 (2): 209-27.

Pretty, J. (2003). Social Capital and the Collective Management of Resources. Science, 302: I 91 2-4.

Putnam, R. (2000). Bowling Alone. The Collapse and Revival of American Community. Nueva York: Simon \& Schuster.

San Martín, G., Parma, A. M., y Orensanz, J. M. L (2010). The Chilean Experience with Territorial Use Rights in Fisheries. En eds. R. Grafton, D. Squires, M. Tait y M. Williams, (eds.), Handbook of Marine Fisheries Conservation and Management. Nueva York: Oxford University Press.

SAnZ, L. (2003). Análisis de redes sociales o cómo representar las estructuras sociales subyacentes. Apuntes de Ciencia y Tecnología, 7: 2I-9.

Schumann, S. (2007). Co-management and «Consciousness»: Fishers' Assimilation of Management Principles in Chile. Marine Policy, 3 I: IOI-I I.

-. (20I0). A Tenuous Triumvirate: The Tole of Independent Biologists in Chile's Co-management Regime for Shellfish. Marine Policy, 34 (I): I33-8.

Schurman, R. A. (I996) Snails, Southern Hake and Sustainability: Neoliberalism and Natural Resource Exports in Chile. World Development 24(II):I695-I709.

ScotT, J. (2000). Social Network Analysis: A Handbook. Londres: Sage.

SUBPESCA (2005). Evaluación técnica y económica del impacto de las Áreas de Manejo y Explotación de Recursos Bentónicos. Valparaíso: Subsecretaría de Pesca, Departamento de Pesca Artesanal.

Ther, F. (2008). Prácticas cotidianas e imaginarios en sociedades litorales. El sector de Cucao, Isla Grande de Chiloé. Chungará, 40 (I): 67-80.

Thiel, M., Macaya, E. C., Acuña, E., Arntz, W. E., Bastías, H., Brokordt, K., Camus, P. A., Castilla, J. C., Castro, L. R., Cortés, M., Dumont, C. P., Escribano, R., Fernández, M., Gajardo, J. A., Gaymer, C. F., Gómez, I., González, A. E., González, H. E., Haye, P. A., Illanes, J. 
E., Iriarte, J. L., Lancellotti, D. A., Luna-Jorquera, G., Luxoro, C., Manríquez, P. H., Marín, V., Muñoz, P., Navarrete, S. A., Pérez, E., Poulin, E., Sellanes, J., Sepúlveda, H. H., Stotz, W., Tala, F., Thomas, A., Vargas, C. A., Vásquez, J. A., y Vega, J. M. (2007). The Humboldt Current System of Northern and Central Chile: Oceanographic Processes, Ecological Interactions and Socioeconomic Feedback. Oceanography and Marine Biology: An Annual Review, 45: 195-344.

VAn DER GAAG, M., y Webber, M. (2008). Measurement of Individual Social Capital. En I. Kawachi, S. V. Subramanian, y D. Kim (eds.), Social capital and Health (29-49). Nueva York: Springer.

Wade, R. (1987). The Management of Common Property Resources: Collective Action as an Alternative to Privatisation or State Regulation. Cambridge Journal of Economics, II: 95-106.

Wasserman, S. y Faust, K. (1994). Social Network Analysis: Methods and Applications. Cambridge: Cambridge University Press.

Woolcock, M. (200I). The Place of Social Capital in Understanding Social and Economic Outcomes. Isuma, 2 (I): I I-I 7.

\section{Sobre los autores}

Andrés Marín pertenece al Centro de Conservación Marina del Departamento de Ecología de la Pontificia Universidad Católica de Chile y al Stockholm Resilience Centre de Stockholm University, Suecia. Su correo electrónico es <andres. marin.r@gmail.com>. STEFan Gelcich pertenece al Centro de Conservación Marina del Departamento de Ecología de la Pontificia Universidad Católica de Chile y al Laboratorio Internacional en Cambio Global, Esporles, España. Su correo electrónico es <sgelcich@bio.puc.cl>. Los autores agradecen a los dirigentes de los sindicatos de pescadores artesanales que accedieron a compartir su tiempo y conocimiento. Al proyecto Fondecyt I I 20103 y al Centro de Conservación Marina, iniciativa Milenio Pıo-033F del Ministerio de Economía, Fomento y Turismo, y a la Cátedra de Investigación de Canadá en manejo de recursos basado en la comunidad de Fikret Berkes (Canada Research Chair in Community Based Resources Management). Agradecemos los comentarios y discusiones con Juan Carlos Castilla que ayudaron a mejorar sustancialmente este manuscrito. 
